\title{
Philosophiques
}

\section{Chronique de la vie philosophique}

Volume 2, numéro 2, octobre 1975

URI : https://id.erudit.org/iderudit/203040ar

DOI : https://doi.org/10.7202/203040ar

Aller au sommaire du numéro

Éditeur(s)

Société de philosophie du Québec

ISSN

0316-2923 (imprimé)

1492-1391 (numérique)

Découvrir la revue

Citer ce document

(1975). Chronique de la vie philosophique. Philosophiques, 2(2), 377-389.

https://doi.org/10.7202/203040ar d'utilisation que vous pouvez consulter en ligne.

https://apropos.erudit.org/fr/usagers/politique-dutilisation/ 


\section{CHRONIQUE}

\section{de la vie philosophique}

\section{CONGRÈS}

L'Association Canadienne-française pour l'avancement des Sciences (ACFAS) a tenu cette année son $43 \mathrm{e}$ congrès à l'Université de Moncton du 6 au 9 mai 1975. C'est la Société de Philosophie du Québec qui s'est chargée, à cette occasion, de l'organisation du secteur de la philosophie en y tenant son deuxième congrès annuel. Les travaux furent divisés en communications et en ateliers.

Les communications suivantes furent présentées: P. McCormick, (Ottawa), L'Esthétique de Diltbey: Phénoménologie et théorie littéraire, F. M. Denis (Collège de Maisonneuve), Présentation d'un cadre expérimental d'analyse du contenu (CAXAC) appliqué aux textes pbilosopbiques, R. Lambert, (UQAM), L'idéologie de la rationalité, $M$. Vernet (Queen's Un. Kingston), Travail sur l'idéologie et travail de l'idéologue, L.P. Bordeleau (Ottawa), Maurice Blondel, un réformateur social, V. Mendenhall (Ottawa), La conscience esthétique chez Jean Nabert, G. Lafrance (Ottawa), Le concept de moralité dans la sociologie durkheimienne, P. Gravel (Un. de Montréal), Remarques en biais en vue d'une théatrologie sur la Poétique d'Aristote, T.F. Geraets (Ottawa), Sens perçu, profondeur et réalité dans la Phénoménologie de la perception, R. Pellerin (Col. Laflèche, TroisRivières), L'acte gratuit et l'affirmation de soi par l'intérêt propre, R. Brouillet (Un. Laval, Québec), Dieter Henrich et «The proof-structure of Kant's transcendental deduction $\gg$ Réflexions critiques, J. Philippoussis (Dawson Col. Montréal), Quelques réflexions sur la dramaturgie grecque et la personne bumaine, J. Theau (Ottawa), Remarques sur l'épistémologie française et l'épistémologie américaine, M. Renault (UQTR), La décision 
ontologique, C. Panaccio (UQTR), Sur l'équivocité de la notion de jugement de valeur, W. Villeneuve (Un. Laval, Québec), Alexandre d'Apbrodise, pbilologue.

Quant aux ateliers et tables-rondes, ils furent consacrés à «La Philosophie du Droit» et à «L'analyse des idéologies».

L'Association Canadienne de Philosophie a tenu son dixneuvième congrès annuel à l'Université d'Alberta, Edmonton, du 4 au 7 juin 1975. $\bar{A}$ cette occasion les communications suivantes furent présentées: F. Duchesneau (Ottawa) : Problèmes relatifs à la notion lockienne, d'idée, L. Valcke (Sherbrooke): Rationalisme de Bachelard versus rationalisme classique, N. Lacharité (UQAM) : Vers une typologie des discours non-épistémologiques sur la science, L. Raphaël (Concordia) : Hertz: son rapport à Wittgenstein, O. Reboul (Montréal) : Remarques sur le langage et l'idéologie, Jean Roy (Montréal): Utopie et modernité, E. James Crombie (Dalhousie): Sur l'arbitraire du concept d'espèce biologique, G. Lafrance (Ottawa) : La contribution de Marcel Mauss à l'antbropologie structuraliste, L. Giroux (UQTR) : L'bistorialité chez Heidegger et son rapport à la philisophie de la vie de Dilthey, Louise Marcil-Lacoste (McGill) : Notes sur le concept d'immutabilité, ou question bizarre sur une banalité, Donald McDonell (Ottawa): L'énoncé: pouvoir de vérité, Simonne Plourde (UQ Rimouski): L'espérance bumaine dans l'cuvre de Gabriel Marcel, Danièle Letocha (Collège de Rosemont) : Culture : le donné et le construit, Robert Nadeau (UQAM) : A propos de l'opposition Nature/Culture, Jean-Paul Brodeur (UQAM) : A propos du dernier livre de Michel Foucault: Surveiller et punir, Yvon Lafrance (Ottawa) : La critique de la philosopbie de l'État de Hegel par Marx (Parag. 261 à 274), Maurice Carignan (Ottawa) : L'extérieur et l'intérieur chez Kierkegaard, Louis-Émile Blanchet (Laval) : Traditions juive et arabe sur l'infini, Réjane Bernier (Montréal): L'explication de l'ontogénèse du vivant, Henri-Paul Cunningham (Laval) : L'impact de l'esprit technologique sur l'bomme contemporain, Théodore F. Geraets (Ottawa) : Dialectique et interrogation, Jean Theau (Ottawa) : Alain et sa philosophie de la religion, Herman F. Suligoj (Humber College) : Les états altérés de conscience: vers une philosophie contemporaine du mysticisme. 


\section{LA SOCIÉTÉ DE PHILOSOPHIE DU QUÉBEC}

La Société a publié durant l'année scolaire 1974-1975 les quatre numéros prévus de son Bulletin. Le contenu du premier numéro a déjà été présenté à nos lecteurs (cf. Vol. II, no. 1, p. 169). Outre les informations courantes, on trouvera dans le deuxième numéro du Bulletin (janvier 1975) un document intitulé : La Formation Générale au CEGEP, réponse au Mémoire $d u$ Conseil $d u$ Patronat, rédigé par un groupe de professeurs de philosophie du CEGEP Édouard-Montpetit ainsi qu'un texte expérimental au plan théorique et pédagogique qui fut donné comme partie du cours Phi. 101 au Collège de Maisonneuve et présenté sous le titre: Critique théorique de la notion de «concret ». La rédaction de ce document est due à $\mathrm{M}$. Pierre Turcotte qui travaillait en équipe avec MM. Jean-Guy Daoust et Gilles Gour. Le troisième numéro (mai 1975) contient le Rapport du Président de la S.P.Q. à l'Assemblée générale tenue le 9 mai 1975 à Moncton ainsi que trois contributions : J.P. Brodeur (UQAM), Un colloque sur l'bistoire de la pensée québécoise, G. Leroux (UQAM), Une de perdue, aucune de retrouvée. $A$ propos de l'écriture philosopbique au Québec, C. Panaccio (UQTR), Remarques sur la prétendue «minceur» de l'écriture philosopbique au Québec. Ce dernier texte de C. Panaccio est une réponse à l'article de G. Leroux. Le quatrième numéro (août 1975) reproduit les communications qui ont été faites au Congrès de l'ACFAS à Moncton à l'intérieur de trois ateliers. Le premier atelier était consacré à la Philosophie du Droit: S. Mullet (U. Concordia), Les droits bumains, G. Bourret, G. Ducasse, G. Legault (CEGEP Bois-de-Boulogne), Les fonctions du droit dans la société, R. Lemay (CEGEP Champlain), Réflexions préliminaires sur la fonction du droit. Le second atelier portait sur les grilles théoriques d'analyse des idéologies selon l'approche marxiste: A. Paradis (UQAM), Réflexions sur l'analyse des idéologies, C. Savary (UQTR), Remarques sur le texte d'André Paradis, R. Nadeau (UQAM), Compte rendu des discussions de l'atelier, $\mathrm{D}$. Braybrooke (Dalhousie), Comment mettre à l'épreuve le soupçon selon lequel une croyance est idéologique? Un troisième atelier était consacré à l'enseignement de la philosophie et critique des idéologies : M. Dufour (CEGEP de Maisonneuve), La pbiloso- 
phie, critique et victime de l'idéologie, D. Pelletier, C. Savary (UQTR), Quelques remarques sur l'évolution de la philosopbie au Québec de 1965 à 1975.

\section{LE CERCLE DE PHILOSOPHIE DE TROIS-RIVIÈRES}

A l'occasion de son dixième anniversaire de fondation, le Cercle de Philosophie de Trois-Rivières a publié une histoire de son développement ainsi qu'un bilan complet de ses activités.

Le Cercle a été fondé en 1965 par le Prof. Alexis Klimov qui venait d'arriver d'Europe à Trois-Rivières en 1964. Jeune professeur, récemment embauché par le Centre des Études Universitaires, affilié à l'époque à l'Université Laval, M. Klimov eut l'idée de fonder un Cercle de Philosophie qui permettrait à des philosophes de toutes tendances de venir exposer publiquement leurs idées et d'engager avec l'auditoire un véritable débat. Le succès du cercle fut immédiat : il attira dès la première année des auditoires de trois cents personnes au rythme d'un conférencier par semaine. Les conférences avaient lieu au Pavillon Tessier de l'Université jusqu'en 1967, puis à partir de cette date au Centre culturel de Trois-Rivières, nouvellement construit. Le Cercle au fil des années acquit une réputation enviable à travers le Québec et tout le Canada par le nombre et la qualité des conférenciers qui y furent invités et par la variété des sujets abordés. En septembre 1972, à Montréal, il a été reconnu comme membre de l'Association des Sociétés de Philosophie de langue française et ses activités annuelles sont publiées dans le Bulletin de la Société de philosopbie du Québec ainsi que dans les Études Philosophiques, revue fondée par Gaston Berger sans compter l'appui toujours fidèle que lui ont donné les média d'information de la région trifluvienne. Pendant ces dix années d'existence, le public du Cercle de Philosophie de Trois-Rivières a été des plus diversifié : chaque conférence réunissait des professeurs et étudiants de philosophie, mais aussi des universitaires d'autres disciplines, des employés de bureau, des commerçants, des artistes, des ouvriers, des infirmières et des gens de professions libérales.

Le bilan complet des activités du Cercle pendant ses dix premières années d'existence demeure difficile à résumer. En tout, il s'agit de 156 conférences sur des sujets les plus divers et 
aptes à intéresser un auditoire. Parmi les conférenciers de l'extérieur invités par le Cercle, mentionnons les noms de Maître René Wintgens (Belgique), E. Jarry (Institut Catholique de Paris), G. Thibon (Paris), J. de Finance (Rome), G. Gusdorf (Strasbourg), N. Sarraute (Paris), P. Devambez (Musée du Louvre, Paris), G. Mialaret (Caen), C. Sacheri (Buenos-Aires), N. Oikonomidès (Athènes), $\mathrm{O}$. Silla (Dakar), $\mathrm{H}$. de Clèves (Louvain), J.C. Piguet (Saint-Gall), A. Hamman (Rome), M. Bruézière (Paris), P. Aubenque (Paris), J. de Monléon (Paris), P. Warren (Le Caire), S. Fleury (Paris), A. Kastler (Prix Nobel de Physique), C. Dédéyan (Paris), S. Farcason (Bucarest), J. Vuillemin (Paris), M. Dufresne (Paris), R. Garaudy (Paris).

\section{LA SOCIÉTÉ DE PHILOSOPHIE DE L'OUTAOUAIS}

La Société de Philosophie de l'Outaouais sous la présidence du Prof. Léon Charette a reçu les conférenciers suivants: L. Charrette, B. Ouellet (Ottawa-Hull), L'idéologie dans l'éducation (19 mars), M. Carignan (Ottawa), Notes sur la pensée sociale de Kierkegaard (26 mars), V. Mendenhall, P. Pelletier (Ottawa), L'art et la révolution (16 avril), L.P. Bordeleau, A. Vachet (Ottawa), Le rôle social du Philosopbe (14 octobre), F. Duchesneau, J. Thorp, P. Rody (Ottawa), Physiologie et Liberté (11 novembre).

\section{ACTIVITÉS DES DÉPARTEMENTS}

\section{Université Laval}

Monsieur le professeur Guy Godin de la Faculté de philosophie a été renommé le 7 juillet 1975 pour une période de 3 ans directeur de l'Institut Supérieur des Sciences Humaines.

À l'intérieur d'un programme de l'Institut Supérieur des Sciences Humaines intitulé : la mutation récente de la société québécoise 1940-1971, un projet porte sur l'évolution des Facultés de philosophie du Québec. L'équipe de ce projet est composée des personnes suivantes: André Vidricaire (UQAM), Guy Godin (Laval), Guy Bouchard (Laval), Germain Dandenault (Sherbrooke), Jean Langlois (Jésuite), Suzanne Leblanc (UQAM), Sheila Mullett (Concordia), Claude Savary (Trois-Rivières), Louise Marcil Lacoste (McGill), Louis Valcke (Sherbrooke). 
On prévoit pour le printemps 76, la publication de 2 tomes dans les Cahiers de l'ISSH: T. I Matériaux pour l'histoire des Facultés de philosophie du Québec; le T. Il contiendra des critiques et des remarques de personnes ayant vécu la période étudiée.

\section{Université de Montréal}

Monsieur Yvon Gauthier, professeur agrégé au département de philosophie, a participé au 5e Congrès international de logique, méthodologie et philosophie des sciences qui s'est tenu à London (Ontario) du 27 août au 2 septembre 1975. Il y a présenté une communication intitulée: Intuitionnisme et théories matbématiques locales et a présidé une séance de la section «Foundations of mathematical theories».

Monsieur Gauthier a été invité par les départements de mathématiques et de philosophie de l'Université Dalhousie à Halifax pour y donner deux conférences en janvier 1976. Il a été aussi invité par l'École Normale Supérieure de Paris à donner deux conférences au séminaire de mathématiques de $M$. Loi en mai prochain. Ses conférences porteront sur Les fondements critiques de la théorie axiomatique des ensembles et Le constructivisme et les matbématiques contemporaines. Il a aussi été invité à un Congrès international sur «Langage et pensée mathématiques» qui se tiendra les 9-10-11 juin prochain au Luxembourg où il parlera de Langage et pensée dans l'intuitionnisme post-brouwerien.

M. le Professeur Jacques Poulain a participé au colloque sur "Le religieux aujourd'hui », tenu à Cerisy-La-Salle (France) du 19 août au 30 août 1975. Il y a présenté une communication intitulée: Une reconstruction pbilosopbique du concept de Dieu est-elle aujourd'bui possible?

MM. Garbis Kortian et Jean Roy ont participé pour leur part au colloque sur "Le Discours utopique », tenu également à Cerisy-La-Salle du 22 juillet au 1er août 1975.

M. le Professeur Venant Cauchy a donné à l'Université de Poitiers (France) un séminaire sur: Le Scepticisme ancien et l'bistoire de la philosopbie ainsi qu'une conférence à la Société 
de Philosophie de Poitiers sur: Considérations critiques sur lidée d'bistoire de la philosophie (5 décembre 1974). Ã l'Université de Grenoble il donna une conférence sous les auspices de la Société Alpine de Philosophie sur le thème: L'bistoire de la pbilosopbie et critique de l'bistoire (25 février). Il était également l'invité de la Société Romande de Philosophie où il présenta une conférence sur: La Spécificité du langage pbilosopbique (26 février), sujet qu'il reprit à l'Université de Neuchâtel (29 février) et à l'Université de Lausanne (28 février). Comme invité de la Société de Philosophie de Bordeaux, il donna une conférence sur: La Pbilosopbie au Québec (7 mars). À l'Université de Toulouse-Le-Mirail, il donna un séminaire pour le groupe de Psycholinguistique sur: Les applications de l'informatique aux textes philosopbiques (7 avril) et une conférence à la Société de Philosophie de Toulouse sur: Le scepticisme dans l'bistoire de la philosophie (12 avril). De mars à avril 1975, le Professeur Cauchy donna à l'U.E.R. de philosophie de l'Université de Toulouse une série de conférences sur l'Épicurisme ancien aux candidats à l'agrégation.

\section{Université du Québec à Montréal}

Suite à des négociations menées par le département de philosophie et avec l'aide financière des départements de sociologie, d'histoire et de sciences religieuses qui ont consenti à investir une partie de leur budget d'acquisitions, la bibliothèque de I'UQAM vient de se porter acquéreur d'une intéressante collection de livres en histoire et en philosophie de la culture au Québec.

Cette collection est le fruit du travail de M. Yvan Lamonde, professeur au Centre d'études canadiennes-françaises de l'Université McGill, qui a consacré plusieurs années à la monter. Historien et philosophe de formation, $M$. Lamonde prépare une thèse de doctorat sur l'enseignement de la philosophie dans la province de Québec. Il a déjà publié une Historiograpbie de la pbilosopbie au Québec chez l'éditeur Hurtubise en 1972.

La collection groupe en majorité des livres, mais aussi des albums de revues et des publications officielles recouvrant plusieurs domaines de l'histoire de la culture au Québec depuis le XVIIIe siècle, notamment la philosophie. 
Un séminaire de maîtrise, dirigé par $M$. Jean-Paul Brodeur, professeur au département de philosophie de l'UQAM, et portant sur la production théorique au Québec, a déjà commencé à exploiter cette importante nouvelle acquisition de la bibliothèque. Un catalogue préliminaire est disponible au département de philosophie, en attendant celui que la bibliothèque s'apprête à imprimer.

La délégation du département de philosophie au $43 \mathrm{e}$ Congrès de l'ACFAS qui s'est tenu du 7 au 9 mai dernier à Moncton, était composée de MM. Robert Nadeau, qui anima un atelier de recherche sur «Les grilles théoriques pour l'analyse des idéologies », André Paradis, qui présenta à l'intérieur de cet atelier ses Réflexions sur l'analyse des idéologies, Jean-Guy Meunier, qui participa à une table-ronde touchant à la même problématique, et enfin Roger Lambert, qui présenta une communication sur L'idéologie de la rationalité.

M. Fernand Couturier a été, à la demande du Conseil des Universités, dégagé de tâche par le département de philosophie et nommé par le Conseil «responsable des dossiers» auprès du comité conjoint des programmes du Conseil des Universités. Cette affectation, valable jusqu'au 31 décembre, pourrait être reconduite au besoin.

A Western, Ontario, du 27 août au 2 septembre, se tenait le Congrès international de Logique, Méthodologie et Philosophie des sciences. M. Normand Lacharité y a prononcé une conférence sur L'épistémologie et la socio-politique des sciences : leurs constructions respectives de la science comme objet.

M. Jean-Paul Brodeur, professeur au département de philosophie, a prononcé, fin mai, une conférence qui avait pour thème : Un ensemble de codes, le code pénal. C'était à l'occasion du Congrès de l'Association canadienne des recherches sémiotiques.

Monsieur Harel Malouin est le nouveau directeur du département de philosophie de l'UQAM. Son mandat commençant le ler juin 1975 et se terminera le 31 mai 1976 (puisqu'il termine le mandat de son prédécesseur Monsieur Normand Lacharité).

Dans le cadre de son programme de maîtrise, le département de philosophie de l'UQAM a organisé un séminaire de conférence 
sur «Psychanalyse, idéologie, philosophie ». Les conférenciers sont : Michel Larivière (25 septembre) : «Entre psychanalyse et philosophie ». Régime Robin (16 octobre) : «Fantasme et luttes de classe dans la pratique discursive politique ». Lise Monette (30 octobre) : «L'approche psychanalytique du fantasme : valorisation ou résistance à l'imaginaire ». Vincent Mauriello (13 novembre) : «Idéal du moi et perversion». André Paradis (27 novembre) : «Folie et criminalité au XIXe siècle : le cas du Québec et de l'Ontario». Claude F. Lévesque (9 décembre) : «L'inscription du dialogue psychanalytique $»$.

\section{Université de Sherbrooke}

Le professeur Maurice Gagnon était cette année coprésident du Comité du programme du Congrès de l'A.C.P. tenu à Edmonton. $\bar{A}$ ce titre, il était chargé de l'organisation de la section française de ce congrès.

Le directeur du Département de philosophie, le professeur Jacques Plamondon, a été nommé doyen de la Faculté des arts et est entré en fonction au milieu de l'été. Il a été remplacé par M. Maurice Gagnon au poste de directeur qu'il occupait, et le professeur Laurent Giroux, de l'Université du Québec à TroisRivières, le remplace comme professeur au Département.

Deux initiatives particulières méritent d'être signalées au Département, avec la reprise de l'année académique : la collaboration de l'un des professeurs avec le Département de psychiatrie du Centre Hospitalier Universitaire, en rapport avec la psychiatrie phénoménologique; et un cours nouveau, intitulé «Le pluralisme philosophique», où les professeurs sont invités à exposer et à discuter, en présence d'étudiants et de leurs collègues, J'orientation générale de leur option fondamentale en philosophie et leurs vues sur le pluralisme.

Le programme des activités de la Société de philosophie de Sherbrooke, pour le premier semestre, comporte quatre conférences : Laurent Giroux (Sherbrooke); Propos sur une pbilosophie de l'bistoire: Heidegger et Dilthey; historialité et philosophie de la vie (25 septembre), Le Professeur Jean-Paul Brodeur (Collège de Sherbrooke); De l'orthodoxie en philosopbie (23 
octobre), Maurice Gagnon (Sherbrooke), Joseph Tchao (Sherbrooke), Marc St-Laurent (Séminaire de Sherbrooke); Faut-il brûler Marx? (20 novembre), Alexis Klimov (Trois-Rivières); Le pop-art ou le repos des Parques (11 décembre).

\section{Université d'Ottawa}

La Faculté a reçu cette année trois nouveaux professeurs: le Prof. W.H. Dray qui enseignait auparavant à l'Université de Trent, le Prof. N. Sheman, diplômée de l'Université de Harvard et le Prof. J. Thorp, diplômé de l'Université d'Oxford. Par ailleurs, les professeurs C. Stroick, L. Charette et G. Charron jouissent cette année d'un congé sabbatique. M. Jean Theau a été promu professeur titulaire et M. P. McCormick, professeur agrégé.

M. le Professeur Edmond Ortigues a été le professeur invité pour le premier semestre de cette année. Directeur de l'U.E.R. de philosophie de l'Université de Rennes, le professeur Ortigues est l'auteur de: Le Discours et le Symbole et de : L'CEdipe africain. Son cours a porté sur: "Trois études d'anthropologie sur le mal, la religion, la personnalité ».

Dans le cadre de Colloquium, comité qui s'occupe à la Faculté d'inviter des conférenciers, la Faculté a reçu le Prof. H.R. Harré, professeur de philosophie des sciences à l'Université d'Oxford, qui a donné une conférence sur The Ethnogenic Revolution in the Social Sciences ( 6 octobre). Par ailleurs, le Professeur Jean Ladrière (Louvain) a donné une conférence sur L'épistémologie des sciences bumaines (27 octobre) et le Professeur André Côté (Laval) sur Quelques aspects moins connus et méconnus de la Logique d'Aristote (14 novembre).

Un colloque bilingue sur Foi religieuse et épistémologies contemporaines a été organisé par le Prof. François Duchesneau dans le cadre des Conférences McMartin le 24 et le 25 octobre. Le programme comportait quatre conférences: D. Evans (Toronto), Philosophical Analysis and Religious Faith, J. Ladrière (Louvain), Les structures de l'existence et la question du lieu de la foi, L. Dupré (Yale), Religious Faith and Marxist Ideology, P. Breton (Paris), Critique des idéologies et crise de la foi. Ont 
participé aux discussions les professeurs : R. Bellemare (St. Paul), V. Décarie (Montréal), B. Garceau (Ottawa), R. Lapointe (St. Paul), P. Lucier (Montréal), A. McKinnon (McGill), K. Nielsen (Ottawa), E. Ortigues (Rennes), J. Poulain (Montréal), C. Prado (Queen's), M. Renault (UQTR) et A. Shalom (McMaster).

Le Prof. J. Wojciechowski a collaboré à l'ouvrage intitulé : Science and Society, ed. N.H. Steneck, The University of Michigan Press, 1975, avec une étude sur: The Ecology of Knowledge (p. 258-302). Il a donné une conférence sur Knowledge and the World of the Future à Tempo Research Institute (Washington) qui est un centre de recherche organisé par la compagnie General Electric. À l'Université René Descartes (Paris V), le Prof. J. Wojciechowski a donné une conférence sur Le problème de la classification des savoirs dans le cadre du séminaire sur la Sociologie de la science.

Le Prof. Jean Theau a donné une communication portant sur: Le Temps de la mécanique classique, sa structure et sa genèse rationnelle au Congrès international tenu à London, Ontario, du 27 août au 2 septembre 1975, sur la "Logique, méthodologie et philosophie des sciences ». La communication du Prof. Theau sur: La place du nombre dans la dialectique bégélienne et la dialectique bamelienne ainsi que celle du Prof. Geraets sur Dialectique et Interrogation, communications faites au dernier congrès sur Hegel à Moscou, ont été publiées dans l'HegelJarhbuch de 1974 aux éditions Pahl-Rugenstein.

Le Prof. Peter McCormick a participé au Congrès International de Varsovie (Pologne) sur « La critique marxiste de la Phénoménologie et la Philosophie de R. Ingarden » en juin 1975. Il a donné une communication sur: Ingarden on the existence of esthetic objects.

M. le Professeur François Duchesneau a participé au «Locke Workshop » tenu à l'Université de l'Alberta à Edmonton du 3 au 5 octobre 1975. $\grave{A}$ cette occasion il donnait une communication sur le thème suivant: Analysis of Ideas and Knowledge of Phenomena in Locke's Essay. En tant que codirecteur de Dialogue, le Professeur Duchesneau a aussi profité de son séjour de recherches à Paris, pendant l'été, pour rencontrer les directeurs de 
diverses revues françaises telles que Revue de Métapbysique et Morale, Etudes Pbilosopbiques, Archives de Philosophie, Revue Philosopbique de France et de l'étranger, etc. dans le but d'établir des relations de collaboration entre la revue canadienne qu'il dirige et les revues françaises de philosophie.

M. le Professeur Yvon Lafrance a fait paraître le quatrième tome de la collection «L'Univers de la Philosophie » sous le titre : La Pbilosopbie et les Savoirs aux Éditions Bellarmin à Montréal. Les études de ce tome ont été réunies par les Professeurs Robert Nadeau et J.-P. Brodeur de l'Université du Québec à Montréal.

\section{PUBLICATIONS}

M. le Professeur Léonce Paquet vient de publier un ouvrage qui s'intitule: Les Cyniques Grecs. Fragments et témoignages. Il s'agit du quatrième tome publié dans la collection «Philosophica », dirigée par le Professeur Jean-Louis Allard. La publication a été assurée par les Éditions de l'Université d'Ottawa. L'ouvrage du Professeur Paquet contient des textes des cyniques grecs qui n'avaient encore reçu aucune traduction en français.

Dans la collection «Textes et Études Slaves», M. le Professeur Alexis Klimov a publié une anthologie de textes critiques sous le titre: Dostö̈evski miroir aux Presses de l'Université, du Québec. L'ouvrage contient un ensemble d'études sur les principales œuvres de l'écrivain russe.

Également aux Presses de l'Université du Québec, Mlle Simone Plourde vient de publier un ouvrage sur: Gabriel Marcel, pbilosophe et témoin de l'espérance.

Le contenu des Actes du premier colloque de l'Institut d'Études Médiévales tenu à Montréal en avril 1974 vient d'être publié sous le titre: Aspects de la Marginalité au Moyen-Âge. M. le Professeur Guy-H. Allard assuma la direction de cette publication aux Éditions de l'Aurore à Montréal. L'ouvrage comprend les études suivantes : R. Saint-Jacques, Les mendiants dans l'épopée anglaise du XIVe siècle; $\mathrm{B}$. Chaput, La condition juridique et sociale de l'aliéné mental; C. Sutto, L'image du monde à la fin du Moyen-Age; Bruno Roy, En marge du monde connu: Les races de monstres; $\mathrm{F}$. Gagnon, Le thème médiéval de l'bom- 
me sawvage dains les premières représentations des Indiens d'Amérique; A. Paradis, Les oniromanciens et leurs traités des rêves; Jean Goulet, $U n$ portrait des sorcières du XVe siècle; C. Gagnon, Les alchimistes et les spéculateurs. Cet ouvrage inaugure la collection «Explorations» consacrée aux multiples aspects de la philosophie universelle et des sciences humaines.

Les Éditions du Jour à Montréal ont publié un ouvrage de MM. Jacques Dufresne et Serge Barbeau qui s'intitule: Le 100,000ième exemplaire. Essai sur la magie du nombre. Par ailleurs, M. Jacques Dufresne, professeur au CEGEP d'Ahuntsic et directeur de la revue Critère a fait paraître le numéro 11 (décembre 1974) sous le titre: Croissance et Démesure et le numéro 12 (mai 1975) sur: L'Art de vivre.

Quatre professeurs du CEGEP d'Ahuntsic, MM. François Charbonneau, Marie-José de Groot, Raymond Fredette et JeanClaude Martin viennent de publier un ensemble de fascicules destinés à servir à l'enseignement de la philosophie au niveau collégial. Le tout se présente sous le titre: Outils à penser sous une forme pratique et attrayante. Le fascicule intitulé : Diagnostic présente une étude sur la formation générale des cégépiens. Le fascicule intitulé : Pensées pour penser rassemble les divers textes auxquels renvoient tous les autres fascicules. Les quatorze fascicules d'outils visent à faire comprendre un certain nombre de notions dont l'étudiant aura besoin pour réaliser ses tâches. Les sept fascicules de tâches visent à la réalisation d'un certain nombre de tâches dans le but d'apprendre à mieux penser. Aucun ordre général ne s'impose a priori dans la réalisation des tâches. Il s'agit donc d'un instrument pédagogique souple qui peut s'adapter facilement aux formules pédagogiques de tutorat, de groupe de discussion ou encore à des formules mixtes. On peut se procurer cet instrument pédagogique en s'adressant à : La direction de l'enseignement des Arts et Lettres et des Communications Graphiques, Collège Ahuntsic, 9155, rue St-Hubert, Montréal, Québec. Tél. (514) 389-5921, poste 224. 